\title{
Estudo epidemiológico de cães com imagens radiográficas compatíveis com neoplasias ósseas
}

\author{
Epidemiological study of dogs with compatible image
} of Skeletal Neoplasms by the radiographic examination

\section{Cristiano Gomes', Márcio Poletto Ferreira', Kelly Cristina Rocha da Silva Ferreira², Ruben Lundgren Cavalcanti ${ }^{3}$, Camila Spagnol ${ }^{3}$, Luciana Oliveira de Oliveira ${ }^{4}$, Emerson Antônio Contesini ${ }^{5}$ \& Rosemari Teresinha de Oliveira ${ }^{6}$}

\begin{abstract}
RESUMO
As neoplasias ósseas representam aproximadamente $5 \%$ de todas os tumores que acometem cães e gatos. As neoplasias ósseas ocorrem principalmente em cães de porte grande, de meia idade a idosos, sendo que os machos são mais predispostos. O objetivo deste trabalho é apresentar os aspectos epidemiológicos dos cães com imagem compatível com neoplasia óssea através do exame de radiográfico. Neste estudo foram analisados cães com imagens radiográficas compatíveis com neoplasia óssea atendidos no Hospital de Clínicas Veterinárias da Universidade Federal do Rio Grande do Sul (HCV-UFRGS) no período de janeiro de 2001 a dezembro de 2004. Foram contabilizados neste estudo 67 cães com imagens compatíveis com neoplasias ósseas, dentre os quais houve uma maior incidência em fêmeas $(55,22 \%)$. A raça mais predisposta foram os cães da raça Rottweiler, seguidos pelos cães sem raça definida, Fila brasileiro e Pastor Alemão. A localização mais freqüente dessas imagens foi observada nos membros posteriores (41,79\%) e nos membros anteriores (40,30\%). A idade média foi de 7,9 anos, variando de 2 a 15 anos. A maior predisposição de cães de grande porte e a idade média dos cães confirmaram os relatos da literatura, entretanto foi observada uma maior incidência em fêmeas que em machos e os membros anteriores não foram mais acometidos que os membros posteriores como afirma a literatura.
\end{abstract}

Descritores: neoplasia óssea, câncer, osteossarcoma.

\section{ABSTRACT}

The skeletal tumors represent approximately $5 \%$ of all tumors in dogs and cats. It occurs specially in large breeds, middle-aged to old animals and males are more predisposed. The aim of this paper is to show the epidemiologic aspects in dogs with compatible image of skeletal neoplasms by the radiologic examination. In this work were analyzed dogs which images were compatible to skeletal neoplasms by radiologic examination. They were attended in the Veterinary Hospital of the Federal University of Rio Grande do Sul from January 2001 to December 2004. It was identified 67 dogs with skeletal tumors, occurring in higher incidence in females (55.22\%). It was identified a stronger occurence in Rottweillers, followed by mixed breeds, Fila Brasileiro and German Shepherd. These tumors were often seen in the posterior limb (41.79\%) and in the anterior limb (40.30\%). It was observed that occurrence average is 7.9 years old, varying between 2 to 15 years. The higher incidence of the large breed and the median age of the dogs confirmed the literature report, however it was observed a higher incidence in females than males and in the anterior limbs were not more affected than the posterior limbs as the literature affirm.

Key words: skeletal neoplasm, cancer, osteosarcoma. 


\section{INTRODUÇÃO}

As neoplasias ósseas representam aproximadamente 5\% de todas os tumores que acometem cães e gatos. Estes tumores são geralmente malignos, sendo o osteossarcoma o mais frequiente, acometendo mais de $85 \%$ destes animais $[3,9]$. Entretanto, pode-se encontrar outros tipos histológicos [9]. As neoplasias ósseas ocorrem principalmente em cães de porte grande a gigante [9,11]. Esta enfermidade ocorre principalmente em cães de meia idade a idosos e os machos parecem ser mais predispostos que as fêmeas [9].

As características radiográficas deste tumor são lesões líticas e proliferativas na região metafisária dos ossos longos, aumento de volume dos tecidos moles com calcificação se estendendo a estes tecidos formando espículas periostais (aspecto de explosão solar), a presença do "Triângulo de Codman" que se caracteriza por uma reação óssea entre o periósteo e o córtex, além desta neoplasia raramente atravessar a cápsula articular $[1,3,7,10]$. Estas características dão o embaseamento de neoplasia óssea, entretanto o diagnóstico definitivo e a diferenciação do seu tipo histológico são dados através da histopatologia [9,12]. O diagnóstico diferencial deve ser feito de tumores metastáticos em ossos, micoses sistêmicas com localização óssea e osteomielite bacteriana [1,7].

O objetivo deste trabalho é apresentar os aspectos epidemiológicos dos cães com imagem compatíveis com neoplasias ósseas através do exame radiográfico atendidos no Hospital de Clínicas Veterinária da Universidade Federal do Rio Grande do Sul no período de janeiro de 2001 a dezembro de 2004.
Durante a avaliação dos dados epidemiológicos destes animais foi observada uma maior predisposição para as raças de grande porte, sendo que a raça Rottweiler foi a mais freqüentemente acometida com 21 casos $(31,34 \%)$, seguida por cães sem raça definida (S.R.D.) com 15 casos (32,39\%), conforme demonstra a Figura 1.

Dos 15 cães S.R.D., foi possível registrar o peso de 13 destes animais, sendo observado uma maior predisposição para cães com peso maior que $15 \mathrm{~kg}$ com 10 casos $(76,92 \%)$, quando comparado com cães com peso inferior a $15 \mathrm{~kg}$ com 3 casos $(23,08 \%)$.

Os cães machos tiveram um menor índice quando comparado com as fêmeas. Dos 67 casos de tumores ósseos em cães, 37 (55,22\%) foram em fêmeas e $30(44,78 \%)$ em machos. A idade média dos cães foi de 7,9 anos, variando entre 2 e 15 anos de idade.

As principais localizações desta neoplasia foram nos membros posteriores e nos membros anteriores, conforme Figura 2.

\section{DISCUSSÃO}

Os dados quanto a predisposição racial foram de acordo com os dados encontrados na literatura onde afirmam que esta doença ocorre mais freqüentemente em cães de porte grande a gigante $[1,3,4,6,9,12,13]$.

Quando avaliou-se o peso dos cães S.R.D., foi observado uma maior predisposição para cães com peso acima de $15 \mathrm{~kg}$, confirmando que o tamanho parece ser um fator mais importante de predisposição que a raça $[3,5,7,11]$.

\section{MATERIAIS E MÉTODOS}

Foram avaliados cães atendidos no HCV-UFRGS que ao exame radiológico foi constatado imagens compatíveis com neoplasias ósseas no período de janeiro de 2001 a dezembro de 2004.

Os dados foram analisados quanto a raça, idade, sexo e localização do tumor destes animais, sendo excluídos do trabalho animais com dados clínicos incompletos.

\section{RESULTADOS}

Foram verificados no setor de Radiologia do HCV-UFRGS um total de 67 casos de cães com imagens compatíveis com neoplasia óssea.

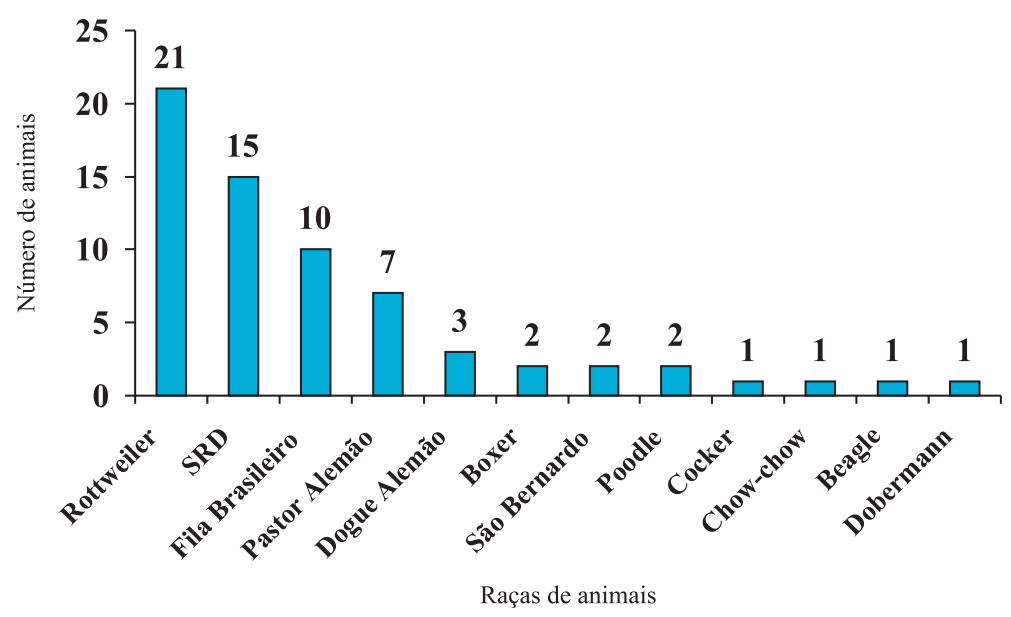

Figura 1. Distribuição racial dos cães com imagens compatíveis de neoplasia óssea através do exame radiográfico atendidos no HCV-UFRGS no período de janeiro de 2001 a dezembro de 2004. 


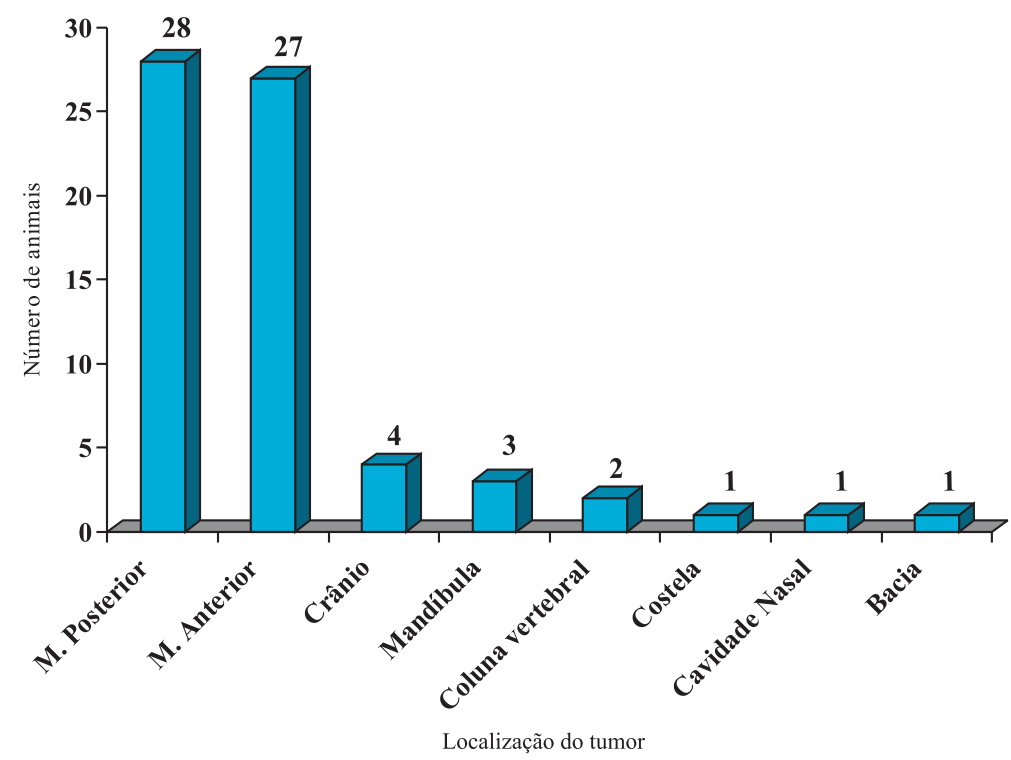

Figura 2. Distribuição da localização das lesões em cães com imagens compatíveis ao exame radiográfico de neoplasia óssea atendidos no HCV-UFRGS no período de janeiro de 2001 a dezembro de 2004. idade a idosos com idade média de 7 anos [3-5,8,11]. Waters e Cooley (1998) e Endicott (2003) ainda afirmam haver uma distribuição em duas fases de 2 e 9 anos de idade, dados que não ficaram evidentes neste trabalho, onde foi observado uma distribuição mais homogênea das idades.

As referências quanto a localização destes tumores são semelhantes aos encontrados na literatura, que relata que $75 \%$ destes tumores ocorrem no esqueleto apendicular [3,4] Entretanto, os dados da literatura afirmam que os membros anteriores são duas vezes mais afetados que os membros traseiros [3,4,9], o que não foi confirmado neste trabalho onde o número de casos comparando os membros foram muito próximos.

\section{CONCLUSÕES}

Entretanto, os dados referentes a predisposição quanto ao sexo vieram de encontro aos dados encontrados na literatura, que afirmam que esta enfermidade ocorre mais freqüentemente em machos $[2,3,5,9,11]$.

A idade média de ocorrência desta doença foi muito próxima aos dados da literatura que afirmam que este tumor ocorre principalmente em cães de meia
Os aspectos epidemiológicos dos cães com imagens compatíveis de neoplasia óssea através do exame radiográfico avaliados neste trabalho confirmaram os relatos da literatura quando a idade média e a predisposição racial, entretanto houve divergência quanto a maior predisposição de fêmeas e a localização do tumor, onde não foi confirmada uma maior incidência desta neoplasia nos membros anteriores.

\section{REFERÊNCIAS}

1 Chun R. \& Lorimier L.P. 2003. Uptade on the biology and management of canine osteosarcoma. The Veterinary Clinics of North America Small Animal Practice. 33: 491-516.

2 Coomber B.L., Denton J., Sylvestre A. \& Kruth S. 1998. Blood Vessel Density in Canine Osteosarcoma. Canadian Journal of Veterinary Research. 62: 199-204.

3 Dernell W.S. 2003. Tumours of the skeletal system. In: Dobson J.M. \& Lascelles B.D.X (Eds). Bsava Manual of Canine and Feline Oncology. 2nd edn. Gloucester: BSAVA, pp.180-191.

4 Endicott M. 2003. Principles of treatment for osteosarcoma. Clinical Techniques in Small Animal Practice. 18: 110-114.

5 Kirpensteijn J., Kik M., Rutteman G.R. \& Teske E. 2002. Prognostic Significance of a New Histologic Grading System for Canine Osteosarcoma. Veterinary Pathology. 39: 240-246.

6 Liptak J.M., Dernell W.S., Ehrhart N. \& Withrow S.J. 2004. Canine Apendicular Osteosarcoma: Diagnosis and Palliative Treatment. Compendium on Continuing Education for the Practicing Veterinarian. 1: 172-182.

7 Liptak J.M., Dernell W.S., Ehrhart N., Withrow S.J., Séguin B., Walsh P.J. \& Kuntz C.A. 2004. Canine Apendicular Osteosarcoma: Curative-Intent Treatment. Compendium of Continuing Education for the Practicing Veterinarian. 2: 186-196.

8 Morello E., Vasconi M. E., Martano M., Peirone B. \& Buracco P. 2003. Pasteurized Tumoral Autograft and Adjuvant Chemoterapy for Treatment of Canine Distal Radial Osteosarcoma: 13 Cases. Veterinary Surgery. 32: 539-544.

9 Morris J. \& Dobson J. 2001. Skeletal system. In: Small Animal Oncology. Oxford: Blackwell Science, pp.78-93.

10 Ogilvie G.K. 2004. Tumores Ósseos. In: Rosenthal R.A. (Ed.). Segredos em Oncologia Veterinária. Porto Alegre: Artmed, pp.183-189. 
Gomes C., Ferreira M.P., Ferreira K.C.R.S., Cavalcanti R.L., Spagnol C., Oliveira L.O., Contesini E.A. \& Oliveira R.T. 2006. Estudo epidemiológico de cães com imagens radiográficas compatíveis... Acta Scientiae Veterinariae. 34: 159-162.

11 Straw R.C. 1996.Tumors of the skeletal system. In: Macewen E.G. \& Withrow S.J. (Eds.) Small Animal Clinical Oncology. 2nd edn. Philadelphia: WB Saunders, pp.287-315

12 Waters D.J. 1998. Sistema Músculo-esquelético. In: Slatter D. (Ed.) Manual de Cirurgia de Pequenos Animais. 2.ed. São Paulo: Manole, pp.2607-2623

13 Waters D.J. \& Cooley D.M. 1998. Skeletal neoplasms. In: Morrison W.B.(Ed.) Cancer in Dogs and Cats: Medical and Surgical Management. Baltimore: Williams \& Wilkins, pp.639-653. 